\title{
Prevalência da tuberculose multirresistente no Estado do Ceará, 1990-1999*
}

\author{
Elizabeth Clara Barroso ${ }^{1}$, Jorge Luís Nobre Rodrigues ${ }^{2}$, Valeria Góes Ferreira Pinheiro ${ }^{3}$, Creuza L. Campelo ${ }^{4}$
}

Tuberculose multirresistente (TBMR) é uma preocupação mundial, mas sua magnitude era desconhecida no Estado do Ceará, região Nordeste do Brasil. Objetivos: Determinar a prevalência da TBMR em nosso meio, bem como a sensibilidade do $\mathrm{M}$. tuberculosis às drogas antituberculose. Método: Estudo retrospectivo foi conduzido utilizando a lista de 1.500 testes de sensibilidade (TS) para rifampicina (RFP), isoniazida (INH), estreptomicina (SM), pirazinamida (PZA), etambutol (EMB) e etionamida (ETH) realizados no Laboratório Central de Saúde Pública do Estado do Ceará (Lacen), no período de 19901999. Foi utilizado o método das proporções. Resultados: Resistência primária ou adquirida a pelo menos uma droga foi observada em 404 de 1.500 (27\%) cepas; 266 (17,7\%) casos foram classificados como TBMR devido à resistência a pelo menos RFP + INH. O total de casos de tuberculose (TB) notificados no Estado no período de estudo foi de 41.073, a prevalência da TBMR foi, então, de 0,65\%. Dos examinados, 62,3\% eram do sexo masculino. A média etária foi de 40,17 $\pm 14,62$. A resistência a uma droga isolada foi de $8,1 \%$, para $\mathrm{INH}=5,7 \%$, SM $=1,5 \%$ e $\mathrm{RFP}=0,9 \%$. A resistência a duas, três, quatro, cinco e seis drogas foi, respectivamente, de 10,$8 ; 3,1 ; 0,9 ; 1,5$ e 2,9\%. A sorologia anti-HIV foi realizada em todos os internados e alguns ambulatoriais, totalizando 86 pacientes. Foi negativa em todos. A probabilidade de sobrevivência em cinco anos (138 pacientes analisados) foi significantemente maior no grupo com TBMR tratado com medicamentos de terceira linha, mesmo para o grupo de falência a essas drogas, comparado com o grupo não tratado com esses fármacos. Conclusão: A prevalência da TBMR nesse estudo foi intermediária, quando comparada com o Brasil e outras partes do mundo. Medidas preventivas, diagnóstico precoce e incentivo à pesquisa de novas drogas efetivas e seguras devem ser estimulados no combate à TBMR.

(J Pneumol 2001;27(6):310-314)

\section{Multidrug-resistant tuberculosis prevalence in Ceará State - Northeastern Brazil, 1990-1999}

Multidrug-resistant tuberculosis (MDR-TB) is a worldwide concern but its magnitude in the state of Ceará - Northeastern Brazil has been so far unknown. Objectives: To determine the prevalence of MDR-TB among us as well as the sesceptibility of Mycobacterium tuberculosis to antituberculosis drugs. Methods: A retrospective study was conducted using 1,500 susceptibility tests (ST) for rifampin (RFM), isoniazid (INH), streptomycin (SM), pyrazinamide (PYZ), ethambutol (ETM) and ethionamide (ETH) that were performed at the Central Laboratory of the State of Ceará (LACEN) between 1990 and 1999. The proportion method test was used. Results: Of the 1,500 stains studied, $404(27 \%)$ were resistant to at least one drug, including both primary and acquired resistance. MDR-TB, defined as resistance to at least rifampin and isoniazid was observed in 266 $(17.7 \%)$ patients. During the study period, there were 41,073 TB cases and MDR-TB prevalence was

* Trabalho realizado no Serviço de Tisiologia do Hospital de Maracanaú, Ministério da Saúde, Fortaleza, CE.

1. Mestranda em Clínica Médica da Universidade Federal do Ceará; Pneumologista do Ministério da Saúde-Hospital de Maracanaú e da Secretaria de Saúde do Estado do Ceará-Hospital de Messejana.

2. Doutor em Doenças Infecciosas e Parasitárias; Chefe da Comissão de Infecção Hospitalar do Hospital Universitário Walter Cantídio.

3. Mestre em Pneumologia; Doutoranda em Farmacologia da Universidade Federal do Ceará; Professora Assistente de Pneumologia, Departamento de Medicina Clínica da Universidade Federal do Ceará.
4. Farmacêutica Bioquímica do Laboratório Central de Saúde Pública do Estado do Ceará (Lacen).

Endereço para correspondência - Elizabeth Clara Barroso, Rua Fonseca Lobo, 50, apto. 402 - 60175-020 - Fortaleza, CE. Tel./Fax (85) 267-1557; E-mail: vbarroso@fortalnet.com.br

Recebido para publicação em 10/5/01. Aprovado, após revisão, em 14/9/01. 
$0.65 \%$ in our state. Among the patients studied, $62.3 \%$ were men and mean age was $40.17 \pm$ 14.62. Overall prevalence for single-drug resistance was $8.1 \%$ (INH $=5.7 \%$; $\mathrm{SM}=1.5 \%$; and $\mathrm{RFP}=$ $0.9 \%$ ). Resistance to $2,3,4,5$ and 6 drugs was, respectively, $10.8 \% ; 3.1 \%$; $0.9 \% ; 1.5 \%$; and $2.9 \%$. HIV testing was performed in all inpatients and some outpatients (total of 86 patients) and revealed that they were all HIV negative. The probability of 5-year survival (138 patients assessed) was greater in the MDR-TB group treated with alternative drugs, including the patients of the failure group. Conclusion: The MDR-TB prevalence rate found in this study is intermediate in comparison with those reported in Brazil and the other parts of the world. Preventive approaches, early diagnosis and the development of effective, safe and relatively inexpensive new drugs should be stimulated.

Descritores - Tuberculose multirresistente. Análise de sobrevivência. Prevalência.

Key words - Multidrug-resistant tuberculosis. Prevalence. Survival probability.

\section{INTRODUÇÃO}

A tuberculose é a mais antiga companheira da humanidade, sempre esperando condições favoráveis para florescer ${ }^{(1)}$. Conflitos e crises econômicas graves podem ter grande impacto na provisão dos serviços de saúde, ocasionando irregular suprimento de medicamentos, deterioração desses serviços e desmoralização dos seus profissionais ${ }^{(2)}$. O controle da tuberculose, que se supunha que ocorreria por volta do fim do século XX, claramente já teve sua data adiada. O crescimento da incidência da tuberculose (TB) e dos casos de tuberculose multirresistente (TBMR) tem sido publicado em todo o mundo. Apesar disso, os serviços de tuberculose no Brasil continuam fracionados e descoordenados. A ameaça da TBMR não deveria ser negligenciada, porque, se os antibióticos e quimioterápicos se tornarem ineficientes, a tuberculose se tornará incurável, como antes de $1944^{(1)}$. O objetivo deste estudo foi determinar magnitude da TBMR em nosso Estado e o perfil de resistência local às drogas antituberculose.

\section{MÉTOdos}

Foi realizado um estudo retrospectivo da suscetibilidade antimicrobiana de cepas de Mycobacterium tuberculosis no Laboratório Central do Estado do Ceará, de 1990 a 1999. Esse serviço é o único a realizar os testes de sensibilidade (TS) do Estado e é supervisionado pelo Centro de Referência Professor Hélio Fraga. Foi usado o meio de cultura de Löwenstein-Jensen. Para o TS foi utilizado o método das proporções com meio sólido e resistência foi definida como crescimento de pelo menos $1 \%$ de colônias sob concentrações críticas das drogas $(0,2 \mu \mathrm{g} / \mathrm{mL}$ para isoniazida, $2 \mu \mathrm{g} / \mathrm{mL}$ para etambutol e $40 \mu \mathrm{g} / \mathrm{mL}$ para rifampicina) e crescimento de pelo menos $10 \%$ de colônias sob concentrações críticas das drogas $(20 \mu \mathrm{g} / \mathrm{mL}$ para etionamida, $100 \mu \mathrm{g} / \mathrm{mL}$ para pirazinamida e $4 \mu \mathrm{g} / \mathrm{mL}$ para
Siglas e abreviaturas utilizadas neste trabalho

GF - Grupo de falência ao tratamento de terceira linha

GN - Grupo não tratado com drogas de terceira linha

GS - Grupo de sucesso com tratamento com drogas de terceira linha

CSL - Grupo curado após tratamento com drogas de segunda linha

BSL - Grupo bacilífero após tratamento com drogas de segunda linha

HIV - Vírus da imunodeficiência humana

INH - Isoniazida

RFP - Rifampicina

TB - Tuberculose

TBMR - Tuberculose multirresistente

TS - Teste de sensibilidade

LACEN - Laboratório Central de Saúde Pública do Ceará

estreptomicina). Definimos TBMR como resistência a, pelo menos, rifampicina (RFP) $e$ isoniazida (INH).

No período de 1990-1999 o LACEN realizou TS de material proveniente de todo o Estado, de casos já suspeitos de resistência bacteriana, oriundos de unidades sanitárias e hospitais, mas no período de julho/1995 a agosto/1996 foram testadas também todas as amostras de escarro positivas para $\mathrm{M}$. tuberculosis provenientes de quatro unidades de saúde da cidade de Fortaleza (capital do Ceará), visando quantificar a resistência primária e adquirida. Essa parte específica do estudo foi a contribuição do Estado do Ceará para a participação do Brasil no Projeto Global de Vigilância à Resistência às Drogas Antituberculose da Organização Mundial da Saúde. Foi considerada TBMR primária quando se identificou bacilo multirresistente proveniente de paciente sem história de tratamento anterior $e$ TBMR adquirida quando se identificou bacilo proveniente de paciente com história de tratamento anterior.

Os pacientes, identificados a partir do TS, assinaram termo de consentimento do Comitê de Ética em Pesquisa da Universidade Federal do Ceará e foi feita tábua de sobrevivência atuarial. Foram comparados grupos de pacientes que usaram: a) Esquema III - considerado pelos autores como drogas de segunda linha (pirazinamida, estreptomicina, etambutol e etionamida), padronizado no país para uso em casos de falência às drogas de primeira 
linha; b) Esquemas com drogas de terceira linha (amicacina, ofloxacina, clofazimina, claritromicina e metronidazol). Foi considerado óbito por tuberculose quando o doente teve óbito hospitalizado em serviço de tisiologia em conseqüência da TB ativa e nenhum outro co-fator foi identificado.

Os dados demográficos foram expressos como média \pm desvio padrão. A determinação da significância estatística da probabilidade de sobrevivência por cinco anos entre grupos, baseado na análise da tábua de sobrevivência atuarial foi calculada usando o teste do qui-quadrado; $\mathrm{p}<$ 0,05 foi considerado significante.

Foram excluídos os pacientes com TBMR identificados na lista de TS do LACEN e que não foi possível localizar para o follow-up. Excluímos também pacientes cujo contato foi perdido durante o período de acompanhamento.

TABELA 1

Prevalência da resistência combinada aos tuberculostáticos no Laboratório Central do Estado do Ceará, 1990-1999

\begin{tabular}{lrr}
\hline & Pacientes & \% \\
\hline Pacientes & 1.500 & 100 \\
Sensíveis & 1.096 & 73 \\
Qualquer resistência & 404 & 27
\end{tabular}

\section{Resistência a pelo menos}

\begin{tabular}{lrr}
\hline Isoniazida & 365 & 24,3 \\
Rifampicina & 284 & 18,9 \\
Streptomicina & 154 & 10,3 \\
Etionamida & 57 & 3,8 \\
Etambutol & 74 & 4,9 \\
Pirazinamida & 59 & 3,9 \\
\hline Monorresistência & $\mathbf{1 2 2}$ & $\mathbf{8 , 1}$ \\
\hline Isoniazida & 86 & 5,7 \\
Rifampicina & 13 & 0,9 \\
Streptomicina & 23 & 1,5 \\
Etionamida & 0 & 0 \\
Etambutol & 0 & 0 \\
Pirazinamida & 0 & 0 \\
\hline Multidroga resistência & & \\
\hline 2 drogas IR & $\mathbf{2 6 6}$ & $\mathbf{1 7 , 7}$ \\
3 drogas IRS & 144 & 9,6 \\
3 drogas IRE & 43 & 2,9 \\
4 drogas IRSZ & 3 & 0,2 \\
4 drogas IRSE & 5 & 0,3 \\
5 drogas IREEtZ & 43 & 0,6 \\
5 drogas IRSEZ & 9 & 0,4 \\
6 drogas IRSEtZ & 6,3 \\
\hline
\end{tabular}

\section{Resultados}

A população do Estado do Ceará, pelo Censo do Instituto Brasileiro de Geografia e Estatística de 1996, era de 6.809.290 habitantes. O total de notificações de TB ativa no período de 1990 a 1999 foi de 41.073 casos. Dos 1.500 TS realizados no período de 1990-1999, 404 (27\%) cepas foram resistentes a pelo menos uma droga, incluindo a resistência primária e adquirida; 266 eram resistentes a pelo menos RFP + INH, portanto, considerados TBMR. A prevalência da TBMR nesse período foi, então, de $0,65 \%$. A Tabela 1 mostra o perfil de resistência encontrado.

O estudo desenvolvido de julho/1995 a agosto/1996 para resistência primária $e$ adquirida revelou prevalência de TBMR primária de 0,6\%, de TBMR adquirida de 3,3\%, enquanto a prevalência da TBMR combinada foi de $1,1 \%$.

A incidência anual da TBMR está representada na Figura 1.

A média de idade foi de 40,17 $\pm 14,62$ e $62,3 \%$ dos casos eram do sexo masculino. Foi oferecido o teste do HIV para quase todos os pacientes internados e para alguns pacientes ambulatoriais (totalizando 86 pacientes) $e$ em todos eles foi negativo.

Do total de casos de TBMR (266) só identificamos prontuários de 159 pacientes (tratava-se de pacientes de todo

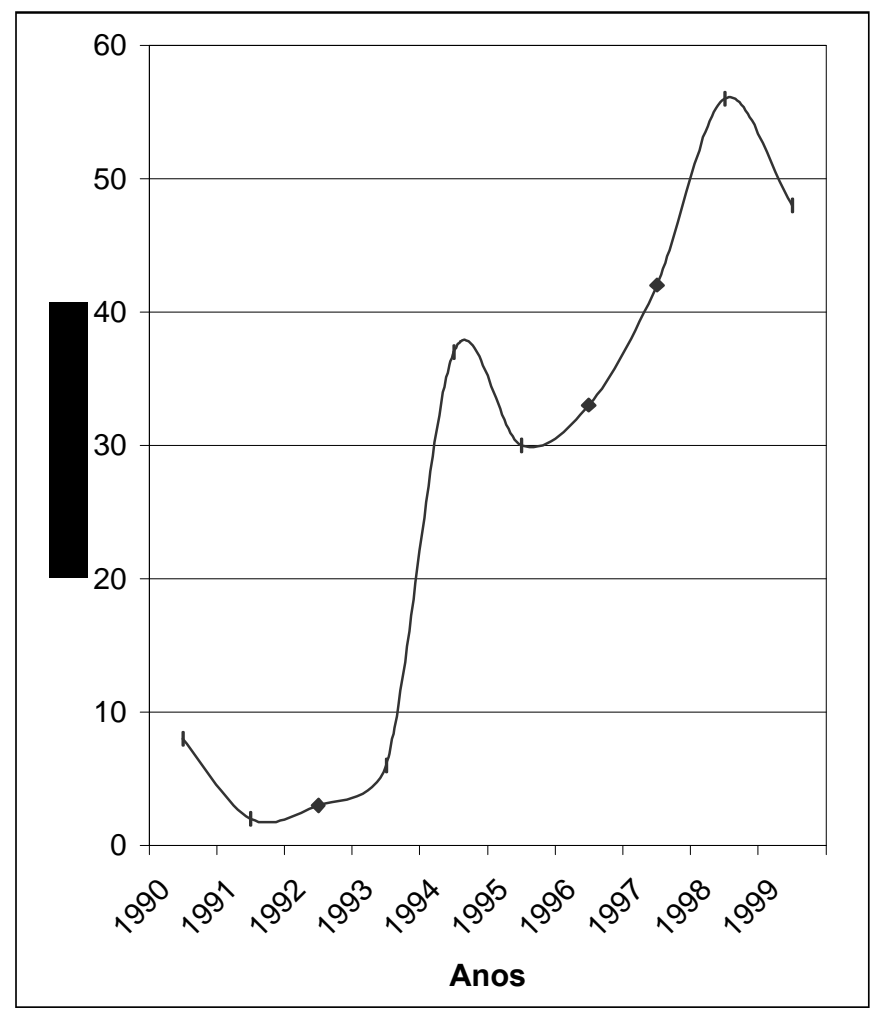

Figura 1 - Incidência anual da tuberculose multirresistente no Estado do Ceará 


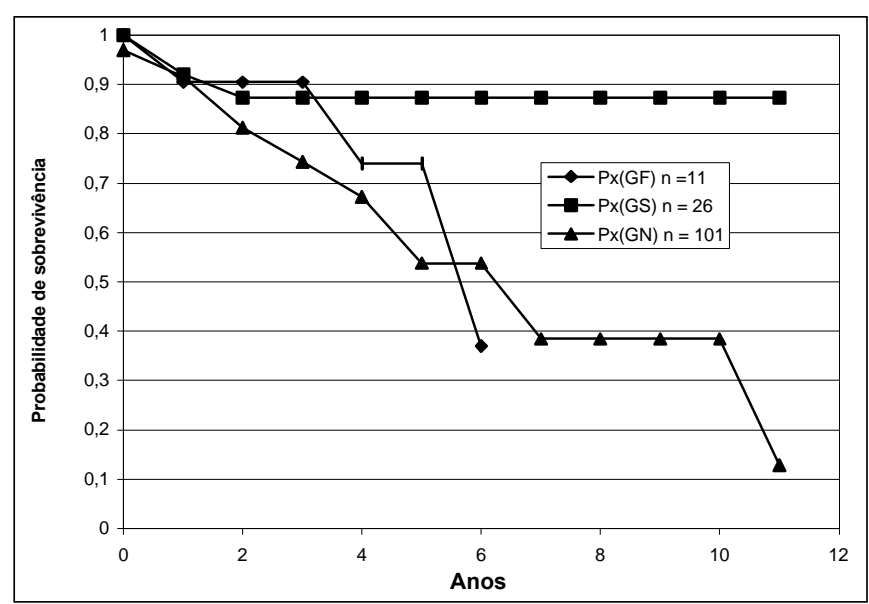

Figura 2 - Tábua de sobrevivência atuarial comparando o grupo de tratamento de sucesso com drogas de terceira linha (GS), o grupo de falência (GF) e o grupo não tratado com drogas de terceira linha (GN). Hospital de Maracanaú, CE.

o Estado). Alguns prontuários não tinham informações suficientes e só pudemos localizar 138 pacientes (em torno de $75 \%$ desses já haviam sido ou vinham sendo acompanhados pelos autores).

A Figura 2 mostra a tábua de sobrevivência atuarial para 138 pacientes com TBMR da data do diagnóstico bacteriológico até a morte ou último follow-up. Os pacientes foram divididos em três grupos. Um grupo $(\mathrm{n}=$ 26) tratado com drogas de terceira linha com sucesso definido como escarro persistentemente negativo à baciloscopia e cultura pelo menos por três meses consecutivos (GS). Um grupo de falência $(n=11)$ que apresentou resposta inadequada pelo menos por seis meses de tratamento (GF). Um terceiro grupo não tratado $(n=101) \mathrm{com}$ drogas de terceira linha (GN). Houve significante diferença na probabilidade de sobrevivência entre os grupos por cinco anos com maior sobrevivência no GS ( $p<0,0019$ ) (Figura 2).

Dos 101 pacientes do GN, 46 curaram-se com o esquema de segunda linha adotado no país. Separando os pacientes curados com drogas de segunda linha $(\mathrm{CSL}=46)$ dos que persistiram bacilíferos com drogas de segunda linha (BSL $=55)$, a probabilidade de sobrevivência diminuiu quase três vezes e a diferença se tornou mais significante ( $p<0,0001$ ) comparando com o grupo de sucesso do tratamento com drogas de terceira linha (Figura 3).

\section{DISCUSSÃO}

Nossa prevalência de TBMR é baixa comparando com o Brasil ou outros países. A prevalência combinada média mundial da TBMR encontrada pelo Projeto Global de Vigilância à Resistência às Drogas Antituberculose, 1994-

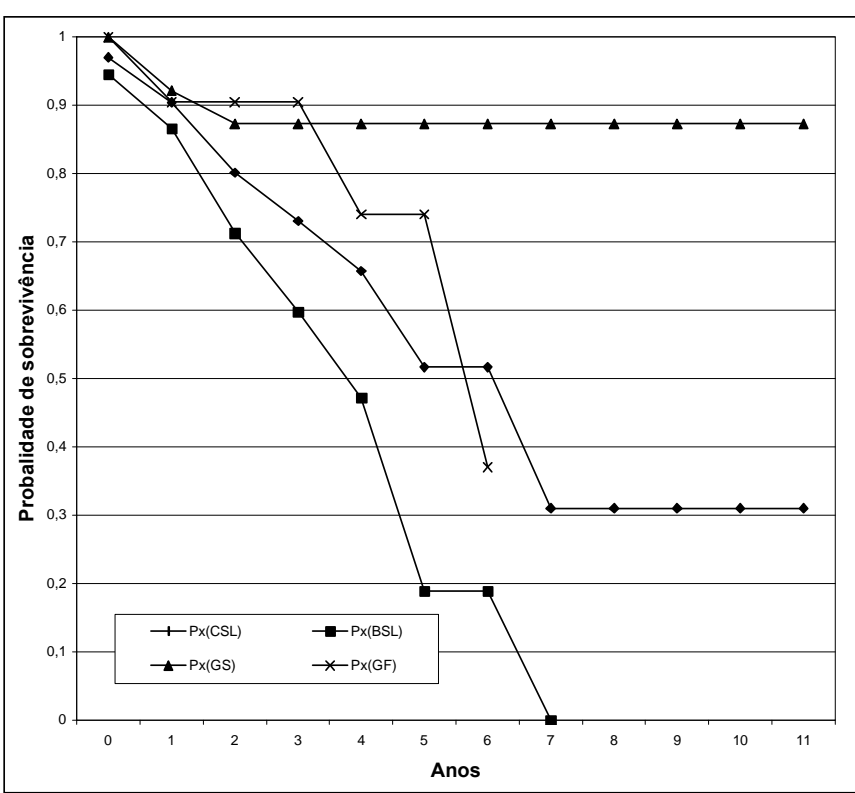

Figura 3 - Tábua de sobrevivência atuarial comparando os grupos da figura 2: aqui o GN foi dividido separando os pacientes que se curaram com as drogas de segunda linha (CSL) e os que persistiram bacilíferos (BSL). Hospital de Maracanaú, $C E$.

$1997^{(3)}$, foi de $5,0 \%$, no Brasil foi de $1,3 \%$ e, no Ceará, de $1,1 \%$.

Nossa prevalência combinada no período de 1990 a 1999 foi de 0,65\%. Embora baixas, nossas taxas são preocupantes devido à tendência crescente que elas vêm mostrando. A prevalência da TBMR em 1994 foi de 0,82\% e aumentou para $1,48 \%$ em 1999 . Numa área rica e industrializada como o Reino Unido, a proporção de TBMR primária aumentou de 0,6\% em 1993 para 1,7\% em $1996^{(4)}$ e, na Alemanha, a TBMR combinada subiu de $1,7 \%$ em 1987 para $5,8 \%$ em $1993^{(5)}$.

Achávamos que o fato de ter sido feita uma pesquisa sistemática para TBMR primária e adquirida no período de julho/1995 a agosto/1996 levaria a aumento no diagnóstico da TBMR nesse período. Mas isso não ocorreu, pois a incidência da TBMR em 1994 já foi um pouco maior que em 1995 (Figura 1).

Análise de sobrevivência tem sido publicada para pacientes imunocompetentes e imunossuprimidos. Numa coorte de 171 pacientes imunocompetentes, Goble et al. ${ }^{(6)}$ publicaram probabilidade de sobrevivência de $87 \%$ para cinco anos para pacientes imunocompetentes com tratamento bem sucedido com drogas de terceira linha. Encontramos a mesma taxa de sobrevivência por cinco anos no grupo com características similares (GS). Park e Da$v_{i s}{ }^{(7)}$ encontraram resultados semelhantes.

Fischl et al. ${ }^{(8,9)}$ atribuíram a alta mortalidade de suas séries ao diagnóstico tardio e falta de tratamento efetivo para TBMR em epidemia nosocomial. Como Park e Da- 
vis $^{(7)}$, gostaríamos de reiterar a importância do diagnóstico precoce e enfatizar que a instituição da terapia adequada tem efeito favorável na duração da sobrevivência.

Uma possível limitação do nosso estudo foi termos localizado apenas 138 pacientes de uma lista de 266 portadores de TBMR; isso poder ter falseado as probabilidades de sobrevivência.

\section{ConClusões}

1) Nossa taxa de TBMR no período de 1990 a 1999 foi baixa, mas preocupante, devido à tendência crescente que apresentou no decorrer do período.

2) Além da crescente incidência anual, a probabilidade de sobrevivência de um paciente com baciloscopia do escarro persistentemente positiva é relativamente longa,

\section{REFERÊNCIAS}

1. March 24, World Tb Day. TB \& HIV 1996;10:2.

2. Glaissberg V. The threat of multidrug resistance: is tuberculosis ever untreatable or uncontrollable? Lancet 1999;353:998-999.

3. Pablos-Mendez A. Global surveillance for anti-tuberculosis-drug resistance, 1994/1997. N Engl J Med 1998;338:1641-1649.

4. Bennett DE, Brady AR, Herbert J, et al. Drug resistant tuberculosis in England and Wales 1993-95. Thorax 1996;51(Suppl 3):S32.

5. Schaberg T, Glober G, Reichert B, Mauch H, Lode H. Resistant lung tuberculosis in Berlin 1987-1993. Pneumologie 1996;50:21-27. o que aumenta a prevalência e a chance de disseminação da doença entre parentes e profissionais de saúde.

3) A co-infecção TB-HIV não está associada ao surgimento de casos TBMR.

4) A instituição de terapia adequada para TBMR aumenta significantemente a sobrevida de pacientes do grupo de sucesso e até mesmo do grupo de falência, em comparação com o grupo sem oportunidade de tratamento.

\section{Agradecimentos}

Os autores agradecem às Assistentes Sociais do Ministério da Saúde Raimunda Oliveira Santos e Adalgisa C.M. Oliveira, lotadas no Hospital de Maracanaú-CE e à Enfermeira Maria S. Ribeiro, da Secretaria de Saúde do Estado do Ceará, pela importante contribuição na realização deste trabalho. Agradecem ainda à Prof ${ }^{a}$ Dra Maria Grasiela Teixeira Barroso, que colaborou em várias fases deste estudo.

6. Goble M, Iseman M, Madsen L, Waite D, Ackerson L, Horsburgh R. Treatment of 171 patients with pulmonary tuberculosis resistant to isoniazid and rifampicin. N Engl J Med 1993;328:527-532.

7. Park M, Davis AL. Outcome of MDR-TB patients, 1983-1993: prolonged survival with appropriate therapy. Am J Respir Crit Care Med 1996; 153:317-324.

8. Fischl MA, Uttamchandani RB, Daikos GL, et al. An outbreak of tuberculosis caused by multiple-drug-resistant tubercle bacilli among patients with HIV infection. Ann Intern Med 1992;117:117-183.

9. Fischl MA, Daikos GL, Uttamchandani RB, et al. Clinical presentation and outcome of patients with HIV infection and tuberculosis caused by multiple-drug-resistant bacilli. Ann Intern Med 1992;117:184-190. 